Table 1. Biofilm Status for KPC-2-Klebsiella pneumoniae Colonizing and Infecting Isolates and Polymyxin B MBEC/MIC Determination for Infecting Isolates

\begin{tabular}{|c|c|c|c|c|}
\hline Patient & Clinical Site & \multicolumn{2}{|c|}{ Biofilm Status } & Polymyxin B MBEC/MIC (Ratio) \\
\hline Patient 1 & Blood & Nonproducing & Nonproducing & $2.0 / 2.0(1)$ \\
\hline Patient 3 & Urine & Weak & Moderate & $8.0 / 2.0(4)$ \\
\hline Patient 4 & Cerebrospinal fluid & Moderate & Moderate & $8.0 / 2.0(4)$ \\
\hline Patient 6 & Blood & Moderate & Strong & $16.0 / 4.0(4)$ \\
\hline Patient 7 & Endotracheal aspirate & Weak & Strong & $128.0 / 8.0(16)$ \\
\hline Patient 8 & Blood & Weak & Weak & $64.0 / 32.0(2)$ \\
\hline Patient 9 & Blood & Weak & Weak & $1.0 / 0.5(2)$ \\
\hline Patient 10 & Urine & Nonproducing & Moderate & $2.0 / 1.0(2)$ \\
\hline
\end{tabular}

Note. MBEC, minimum biofilm eradication concentration; MIC, minimum inhibitory concentration.

2. Rodrigues Perez LR, Dias CG. Emergence of infections due to a polymyxin B-resistant KPC-2-producing Klebsiella pneumoniae in critically ill patients: what is the role of a previous colonization? Infect Control Hosp Epidemiol 2016;37:240-241.

3. Williams DL, Costerton JW. Using biofilms as initial inocula in animal models of biofilm-related infections. J Biomed Mater Res B Appl Biomater 2012;100:1163-1169.

4. Carvalho TS, Perez LR. Impact of biofilm production on polymyxin B susceptibility among Pseudomonas aeruginosa clinical isolates. Infect Control Hosp Epidemiol 2019. DOI: 10.1017/ice.2019.85.

5. Naparstek L, Carmeli Y, Navon-Venezia S, Banin E. Biofilm formation and susceptibility to gentamicin and colistin of extremely drug-resistant KPC-producing Klebsiella pneumoniae. J Antimicrob Chemother 2014;69:1027-1034.
6. Lee MY, Ko KS, Song JH, Peck KR. In vitro effectiveness of the antibiotic lock technique (ALT) for the treatment of catheter-related infections by Pseudomonas aeruginosa and Klebsiella pneumoniae. J Antimicrob Chemother 2007;60:782-787.

7. Perez LRR. Why susceptible bacteria may become resistant to infection control measures? A Pseudomonas biofilm example. Infect Control Hosp Epidemiol 2019;40:386-388.

8. Stewart PS. Mechanisms of antibiotic resistance in bacterial biofilms. Int $J$ Med Microbiol 2002;292:107-113.

9. Andrade LN, Curiao T, Ferreira JC, et al. Dissemination of $b l a_{\mathrm{KPC}-2}$ by the spread of Klebsiella pneumoniae clonal complex 258 clones (ST258, ST11, ST437) and plasmids (IncFII, IncN, IncL/M) among Enterobacteriaceae species in Brazil. Antimicrob Agents Chemother 2011;55:3579-3583.

\title{
Prevalence of bloodstream infections in neutropenic patients with bacteriuria
}

\section{Erica S. Herc MD ${ }^{1,2}$ (1), Rachelle N. Rivera MD³ , Dale Bixby MD ${ }^{4}$ and Carol E. Chenoweth MD ${ }^{5}$}

${ }^{1}$ Division of Infectious Diseases, Department of Internal Medicine, Henry Ford Hospital, Detroit, Michigan, ${ }^{2}$ Wayne State University School of Medicine, Detroit, Michigan, ${ }^{3}$ Renown Health, Reno, Nevada, ${ }^{4}$ Division of Hematology and Oncology, Department of Internal Medicine, Michigan Medicine, Ann Arbor, Michigan and ${ }^{5}$ Division of Infectious Diseases, Department of Internal Medicine, Michigan Medicine, Ann Arbor, Michigan

To the Editor-Patients with hematologic malignancies and neutropenia are at increased risk for several infectious complications. They are predisposed to higher rates of bloodstream infections (BSIs) than the general population. Although BSI is caused by bacteriuria in $\sim 3 \%$ of cases within the general population, ${ }^{1}$ malignancy (odds ratio [OR], 1.94) and receiving immunosuppression within 14 days of bacteriuria $(\mathrm{OR}, 8.13)$ are risk factors for urinary-tract-

Author for correspondence: Erica Herc, Email: eherc1@hfhs.org

Cite this article: Herc ES, et al. (2019). Prevalence of bloodstream infections in neutropenic patients with bacteriuria. Infection Control \& Hospital Epidemiology, 40: 955-956, https://doi.org/10.1017/ice.2019.139 related BSIs. ${ }^{2}$ However, few studies have evaluated the occurrence of secondary BSIs from bacteriuria in neutropenic patients. This study was performed to define the incidence of secondary BSIs in patients with acute myeloid leukemia (AML) with neutropenia and bacteriuria and to determine whether neutropenic patients with asymptomatic bacteriuria have a higher rate of BSI than the general population.

Our study took place in a 900-bed Midwestern tertiary-care hospital with $\sim 2,000$ annual hematology and oncology admissions. We performed a cohort study of all adult patients with AML who were admitted to the hematology service between February 2010 and November 2013. A case was defined as any patient with neutropenia and bacteriuria during the study period. 
Table 1. Prevalence of Findings in Neutropenic Patients with Bloodstream Infection (BSI) Secondary to Urinary Tract Infection

\begin{tabular}{llll} 
& & $\begin{array}{l}\text { Normal } \\
\text { Urinalyses } \\
\text { (Negative } \\
\text { for Leukocyte } \\
\text { Esterase) }\end{array}$ & $\begin{array}{l}\text { Antibiotics That } \\
\text { Covered Pathogenic } \\
\text { Organism Prior to } \\
\text { Cultures }\end{array}$ \\
\hline $\begin{array}{l}\text { Patients with } \\
\text { BSI, No. (\%) }\end{array}$ & $3 / 10(30)$ & $7 / 10(70)$ & $3 / 10(30)$ \\
\hline
\end{tabular}

Bacteriuria was defined by urine culture growth of $>100,000$ colony-forming units per milliliter $(\mathrm{CFU} / \mathrm{mL})$ of a pathogenic organism. Neutropenia was defined by an absolute neutrophil count of $<500$ cells per cubic millimeter $\left(\right.$ cells $/ \mathrm{mm}^{3}$ ) of blood. Secondary BSI was defined by a positive blood culture of the same species as the urine culture within 48 hours of collection of the positive urine culture. Data were collected from the University of Michigan microbiology laboratory and electronic medical records of patients with AML. Patients with bacteriuria were evaluated for the presence of neutropenia and bacteremia. Data collected included demographics, date of first fever if present, date antibiotics were started, antibiotic(s) administered, urinalysis, urine culture results (date of collection and organism), blood culture results (date of collection and organism), and presence of urinary symptoms. The primary outcome was the rate of bacteremia with the same organism (ie, species) as the urine culture within 48 hours of initial collection. Information on microorganism, antibiotic use prior to culture, the presence of urinary symptoms, and the urinalyses were obtained through electronic records using the honest broker. This study was approved by the University of Michigan Institutional Review Board.

In total, 1,807 patients with AML were admitted during the study period. Within the initial cohort, 139 patients $(0.8 \%)$ had bacteriuria. Of the 139 patients with bacteriuria, 102 were neutropenic at the time of culture. Of these patients, 10 of $102(9.8 \%)$ developed bacteremia with the same pathogen as the urine culture. Of 10 isolates, 5 were Enterococcus faecium; it was the most common pathogen that grew in urine and blood cultures within our cohort. Among the E. faecium isolates, 4 of 5 were vancomycin resistant. Other microorganisms isolated included Escherichia coli, Klebsiella pneumoniae, and Enterobacter cloacae. Of 10 patients with BSI, 7 had no documented urinary symptoms. Additionally, 7 of 10 of the patients with BSIs did not receive antibiotics prior to obtaining urine and blood cultures or received antibiotics that did not cover the isolated pathogen. Finally, 7 of 10 urinalyses obtained from patients with bacteriuria and BSIs were negative for leukocyte esterase and pyuria (Table 1).

Approximately $10 \%$ of the patients in our study who were neutropenic with bacteriuria developed bacteremia. A prior study by Krieger et $\mathrm{al}^{3}$ demonstrated that $2.7 \%$ of hospitalized patients within the general population with nosocomial bacteriuria developed BSIs. Most patients in our study who developed bacteremia did not have urinary symptoms documented in their medical record or an abnormal urinalysis; most of the patients who developed bacteremia had no pyuria (Table 1). Patients with neutropenia have a decreased inflammatory response, and pyuria may not occur given a lack of white blood cells; therefore, it is not surprising that most of our patients had no pyuria or leukocyte esterase positivity on their urinalysis.

Current guidelines recommend the use of antibiotics in specific patient populations with asymptomatic bacteriuria including pregnant women, patients undergoing transurethral resection of the prostate, and patients undergoing urologic procedures for which mucosal bleeding is anticipated. ${ }^{4}$ Treatment of asymptomatic bacteriuria in neutropenic patients is not currently recommended. However, as demonstrated in our study, neutropenic patients may not display classic urinary symptoms indicative of a urinary tract infection (UTI) such as dysuria, urinary frequency, or urgency. Additionally, urinalyses in these patients are unlikely to be predictive of a UTI. Of those patients who developed secondary BSIs, $70 \%$ did not receive antibiotics that covered the specific pathogen isolated from urine and blood cultures (prior to the bacteremia). Because the crude mortality rate in patients with BSI and underlying malignancy is between $18 \%$ and $42 \%$, it may be worthwhile to consider treatment in neutropenic patients with asymptomatic bacteriuria to potentially lower the incidence of BSI. ${ }^{5}$

Our study has several limitations, including the small number of patients in our final analysis despite a much larger initial cohort. Additionally, the lack of symptoms documented indicative of a UTI may be due to incomplete documentation in the medical record. Despite these limitations, our study suggests that neutropenic patients with bacteriuria, even without symptoms, may benefit from preemptive treatment. Larger studies are necessary to confirm the findings from our study, which demonstrate that common predictors of UTI were not accurate in our neutropenic patients.

Author ORCIDs. Erica Herc, (iD) 0000-0002-9069-6659

Financial support. No financial support was provided relevant to this article.

Conflicts of interest. All authors report no conflicts of interest relevant to this article.

\section{References}

1. Weinstein MP, Towns ML, Quartey SM, et al. The clinical significance of positive blood cultures in the 1990s: a prospective comprehensive evaluation of the microbiology, epidemiology, and outcome of bacteremia and fungemia in adults. Clin Infect Dis 1997;24:584-602.

2. Saint S, Kaufman SR, Rogers MA, Baker PD, Boyko EJ, Lipsky BA. Risk factors for nosocomial urinary-tract related bacteremia: a case-control study. Am J Infect Control 2006;34:401-407.

3. Krieger JN, Kaiser DL, Wenzel RP. Urinary tract etiology of bloodstream infections in hospitalized patients. J Infect Dis 1983;1:57-62.

4. Nicolle LE, Gupta K, Bradley SF, et al. Clinical Practice Guideline for the Management of Asymptomatic Bacteriuria: 2019 Update by the Infectious Diseases Society of America. Clin Infect Dis 2019;68(10):e83-e110.

5. Wisplinghoff H, Seifert H, Wenzel RP, Edmond MB. Current trends in the epidemiology of nosocomial bloodstream infections in patients with hematological malignancies and solid neoplasms in hospitals in the United States. Clin Infect Dis 2003;36:1103-1110. 